\title{
Editorial: Bridging Environmental Magnetism With Biogeophysics to Study Biogeochemical Processes of Today
}

\author{
Andrea Teixeira Ustra ${ }^{1 *}$, Dimitrios Ntarlagiannis ${ }^{2}$, Leonardo Sagnotti ${ }^{3}$ and Luigi Jovane ${ }^{1}$ \\ ${ }^{1}$ Department of Geophysics, University of São Paulo, São Paulo, Brazil, ${ }^{2}$ Department of Earth and Environmental Sciences, \\ Rutgers University, Newark, NJ, United States, ${ }^{3}$ Istituto Nazionale di Geofisica e Vulcanologia (INGV), Rome, Italy
}

Keywords: magnetic properities, biogeophysics, biogeochemical processes, environment, environmental magnetism

\section{Editorial on the Research Topic}

Bridging Environmental Magnetism With Biogeophysics to Study Biogeochemical Processes of Today

Since the first studies on biogeophysics in the early 2000s our understanding of how biogeochemical processes affect geophysical signatures has significantly improved. This interdisciplinary field now incorporates and integrates environmental and rock magnetism with traditional biological and geochemical methods to interpret geophysical signatures in highly dynamic environments (e.g., biogeochemical hotspots, contamination plumes). Environmental magnetism, in particular, can trace environmental changes by identifying magnetic mineral transformations induced from several biogeochemical processes. This Special Issue includes papers that present applications of environmental and rock magnetism in biogeophysics and discuss their impact on Earth Sciences.

(Bio) geochemical processes are often investigated with environmental magnetism methods in sediments and soils, aiming at identifying changes in the magnetic grain size distribution and stability. For example, microbial magnetite lies in the superparamagnetic (SP) to stable single domain (SSD) size range and variations in the size distribution can trace microbial activities. Ultrafine (SP) magnetic particles in human or animal tissues are also a subject of growing research interest, especially for their environmental significance, and the potential for medical applications. Fine magnetic particles are often observed in the anthropogenic fraction of atmospheric particulate matter, associated with toxic heavy metals, and may be a potential hazard for human health. Information on magnetic grain size distribution is of great value for all these applications.

Hirt and Liu synthesized mixtures of SP and SSD magnetite aiming to estimate their relative contribution in a variety of geological, biological and synthetic samples. The authors showed that both the Day-Dunlop plot and principal component analysis (PCA) analysis of first order reversal curves (FORC) measurements are effective methodologies to detect and quantify changes in the relative proportion of magnetic particles in the SP - SDD state in natural materials and in synthesized particles.

Jaqueto et al. explore the variations of magnetic mineralogy throughout different biomes in South America using a large speleothem rock magnetic database. The authors compare magnetic signals from speleothems, the soil above the cave and the host carbonate rocks to constrain the source and paths of magnetic minerals inside karst systems under different climatic conditions. They recognize that ultrafine ferrimagnetic minerals are the dominant magnetic phase in the speleothems that were studied, with a 
strong correlation between different concentration-dependent magnetic parameters, and that biomes play an important role in the definition of the magnetic content in speleothems.

In a much more dynamic scenario of ongoing hydrocarbon natural degradation, Beaver et al. investigate the microbial community contribution to the observed magnetic susceptibility changes at the National Crude Oil Spill Research Site in Bemidji, Minnesota. The multi-year analysis represents a rich record of microbiology and magnetic changes in hydrocarbon contaminated sediments undergoing biodegradation. The authors show that the highest magnetic susceptibility values were measured in the free-phase petroleum zone, where a methanogenic community was predominant, and not in the overlying ironreducing zone. They hypothesized that this magnetic signature is evidence of the metabolic potential of methanogens to switch their metabolism from methanogenesis to iron reduction with concomitant magnetite precipitation.

Transformations of iron bearing minerals in a trapped gas pocket formed in Quaternary organic sediments is explored by Ustra et al., by tracking specific mineral changes that can be associated to biogeochemical processes. Their investigation is focused on changes regarding the magnetic carrier mineralogy, abundance, and grain size. In addition to classic techniques used in environmental magnetism, the authors utilize quantitative interpretation techniques to characterize the SP mineral content in terms of variations in volume and concentration - along a cored section that intercepts a methane pocket trapped within organic-rich sediments. The authors point out that the association between ironreducing bacteria and SP minerals in a region where both iron reduction and methane accumulation occur could reveal a direct interspecies electron transfer (DIET) scheme.
This Special Issue stems from an engaging session on the topic of biogeophysics and environmental magnetism during American Geophysical Union's Fall Meeting in 2019.

\section{AUTHOR CONTRIBUTIONS}

AU was a guest associate editor of the Research Topic and wrote the editorial text. DN was a guest associate editor of the Research Topic and edited the text. LS was a guest associate editor and acted as editor for two papers in the Research Topic and edited the text. LJ is an associate editor for the Geomagnetism and Paleomagnetism section and edited the text.

Conflict of Interest: The authors declare that the research was conducted in the absence of any commercial or financial relationships that could be construed as a potential conflict of interest.

The handling Editor declared a past co-authorship with one of the authors LJ.

Publisher's Note: All claims expressed in this article are solely those of the authors and do not necessarily represent those of their affiliated organizations, or those of the publisher, the editors and the reviewers. Any product that may be evaluated in this article, or claim that may be made by its manufacturer, is not guaranteed or endorsed by the publisher.

Copyright $\odot 2021$ Ustra, Ntarlagiannis, Sagnotti and Jovane. This is an open-access article distributed under the terms of the Creative Commons Attribution License (CC $B Y$ ). The use, distribution or reproduction in other forums is permitted, provided the original author(s) and the copyright owner(s) are credited and that the original publication in this journal is cited, in accordance with accepted academic practice. No use, distribution or reproduction is permitted which does not comply with these terms. 